\title{
Considerations for Estimating Electrode Performance in Li-Ion Cells
}

\author{
William R. Bennett, NASA Glenn Research Center, Electrochemistry Branch
}

\begin{abstract}
Advanced electrode materials with increased specific capacity and voltage performance are critical to the development of Li-ion batteries with increased specific energy and energy density. Although performance metrics for individual electrodes are critically important, a fundamental understanding of the interactions of electrodes in a full cell is essential to achieving the desired performance, and for establishing meaningful goals for electrode performance. This paper presents practical design considerations for matching positive and negative electrodes in a viable design. Methods for predicting cell-level discharge voltage, based on laboratory data for individual electrodes, are presented and discussed.
\end{abstract}

Index Terms - Energy Storage - batteries.

\section{INTRODUCTION}

$\mathrm{T}$ The proliferation of compact, portable electronic devices has catalyzed continuous improvement in rechargeable battery technologies, driving the development of lithium-ion cells that combine high energy storage with reduced weight (high specific energy) and smaller volume (high energy density). In aerospace applications, such improvements are critical for achieving new missions. Early commercial lithium-ion batteries achieved specific energies of approximately $130 \mathrm{Wh} / \mathrm{kg}$. On-going development, driven by consumer electronics requirements, has resulted in modern, commercial Li-ion cells which achieve specific energies greater than $230 \mathrm{Wh} / \mathrm{kg}$. Recently, Panasonic announced plans to produce 18650-size cells with silicon-based anode materials which reportedly will achieve specific energies of $250 \mathrm{Wh} / \mathrm{kg}$ [1].

Increases in electrode specific capacity are essential for such advances in cell-level specific energy improvements. However, much of the electrode research in the open literature focuses on the performance of individual electrodes, and does not consider the issues associated with performance in full cells.

This paper presents practical considerations for capacitymatching of electrodes in Li-ion cells. A method for estimating cell discharge voltage is described, and the results are used to predict cell level specific energy.

\section{Performance OF SINGLE ELECTRODES IN HALF-CELLS}

Evaluations of single electrodes are performed in half-cells with lithium metal counter-electrodes. Voltage limits for formation and cycling are commonly based on anticipated maximum useable potential windows for the electrode materials under study. These potential windows represent the full utilization (maximum specific capacity) of the electrode material. Capacity matching, and the choice of positive-tonegative $(\mathrm{P} / \mathrm{N})$ ratio, limits the useable electrode potential window in full cells. This will be illustrated in the example, which follows.

\section{A. Typical Electrode Data}

Screening of lithium-ion electrodes is usually performed in half-cells with lithium metal counter-electrodes having a substantial excess capacity relative to the electrode under study. The lithium counter-electrode serves as a pseudoreference electrode, providing data for the individual electrode voltage versus lithium. Laboratory data for the first three cycles of practical, state-of-the-art positive and negative electrodes appears in Fig. 1.

Fig. 1 presents electrode potential as a function of the cumulative capacity (a running total of the charge capacity minus the discharge capacity over several cycles). On charge, the positive electrode is de-lithiated and becomes more positive. At the same time, the negative electrode accepts lithium ions on charge and becomes more negative. On discharge, the process is reversed. For both electrodes, the total first charge capacity is noticeably greater than the discharge capacity. The difference between cumulative charge and discharge capacity is defined as the irreversible capacity of the electrode. The reversible capacity is the capacity that is available to the load after the electrode is formed. The total capacity absorbed by the electrode is the sum of the reversible and irreversible capacity.

For the positive electrode in Fig. 1, a cumulative total capacity of $183 \mathrm{mAh} / \mathrm{g}$ is absorbed in the first few cycles. The reversible capacity is $153 \mathrm{mAh} / \mathrm{g}$. The irreversible capacity of $30.1 \mathrm{mAh} / \mathrm{g}$ is equivalent to $19.7 \%$ of the reversible capacity. 

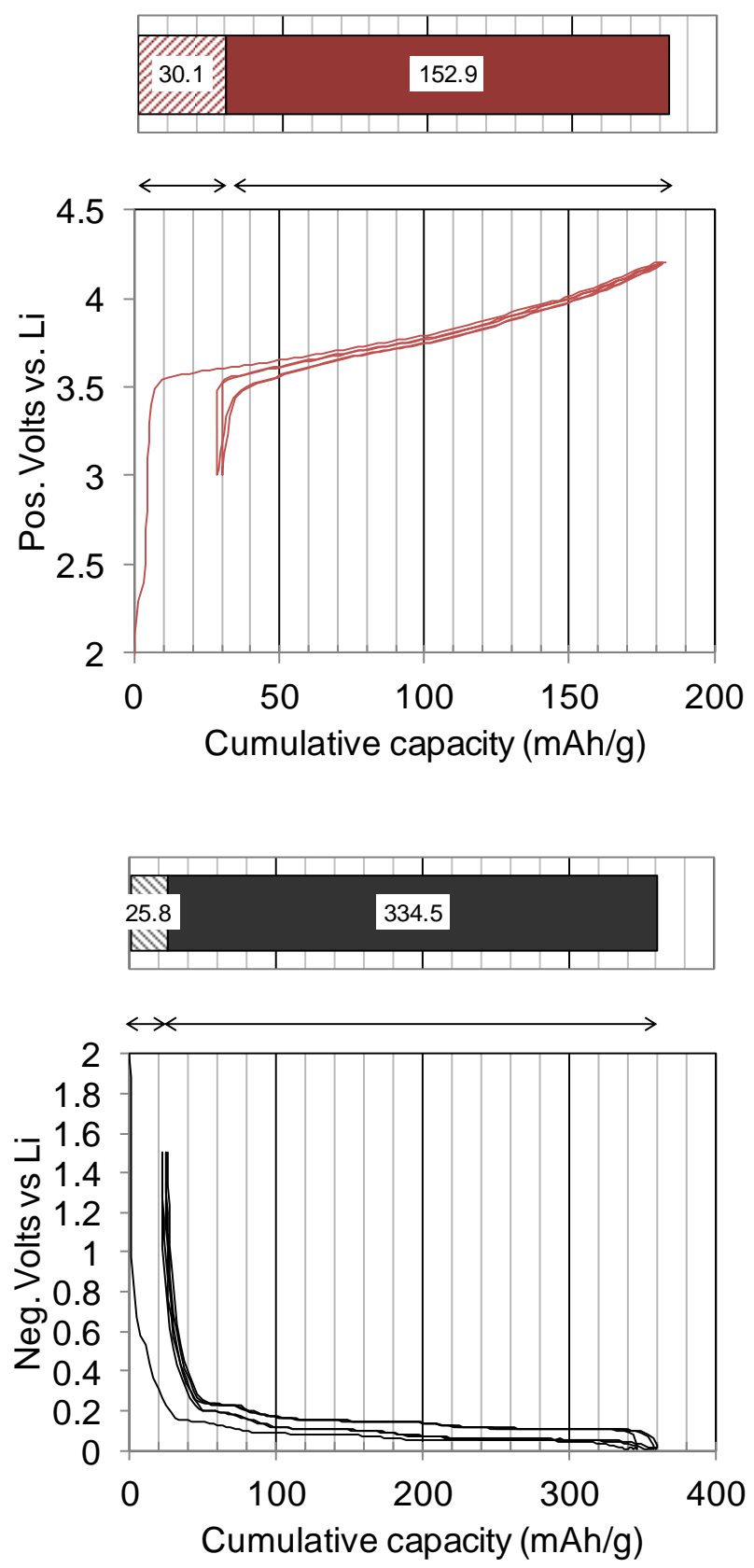

Fig. 1. The first three charge/discharge cycles of a positive and negative electrode in half-cells with lithium metal. Electrode potential versus specific capacity in $\mathrm{mAh}$ per gram of active material. Data at room temperature.

Similarly, the negative electrode absorbs a cumulative charge capacity of $334 \mathrm{mAh} / \mathrm{g}$. The irreversible capacity of 26 $\mathrm{mAh} / \mathrm{g}$ is equivalent to $7.7 \%$ of the reversible capacity. The specific capacity for the Fig. 1 electrodes is summarized in Table 1.
Table I

Specific capacities of electrodes in $\mathrm{mAh} / \mathrm{g}$

\begin{tabular}{ccc}
\hline & positive & negative \\
irreversible & 30.1 & 25.8 \\
reversible & 152.9 & 334.5 \\
\cline { 2 - 3 } total & 183.0 & 360.3 \\
irreversible (\% of rev) & $19.7 \%$ & $7.7 \%$ \\
\hline
\end{tabular}

The reversible specific capacity is used to compute the electrode active material mass that is necessary to meet a desired electrode capacity. When performing cell-level calculations for capacity-balanced electrodes, the specific capacity $(\mathrm{mAh} / \mathrm{g})$ is replaced by total capacity $(\mathrm{Ah})$. When expressed as a fraction of the reversible capacity, the irreversible capacity has the same value regardless of the capacity units.

\section{CAPACITY MATCHING}

The positive and negative electrodes in a practical cell must have essentially equal active area and, exchange capacity with each other during charge and discharge. In state-of-the-art Liion cells, the positive electrode serves as the source of lithium ion. The negative electrode receives lithium from the positive electrode during the first and subsequent charges. A portion of the lithium absorbed by the negative electrode is captured as irreversible capacity, and cannot be returned to the positive electrode. Hence, the reversible and irreversible capacities of the electrodes dictate the useable capacity that is available in the full cell.

\section{A. Reversible Capacity}

The reversible capacity represents the stable capacity that is delivered by the electrode, after the formation cycles are completed. For practical electrodes, the reversible capacity stabilizes after several formation cycles and the cumulative irreversible capacity reaches a limiting value. This is the behavior demonstrated in Fig. 1. In poor-performing electrodes, the irreversible capacity may persist in prolonged cycling, leading to continued loss of cell capacity and poor cycle life. The calculations discussed in this report assume that the capacities are stable after formation.

\section{B. Irreversible Capacity}

Irreversible capacity contributes to the total charge capacity that is absorbed by the electrode in the initial (formation) cycles. In the case of the positive electrode, the extraction of lithium in the first charge induces changes to the crystal structure, making it impossible for the same amount of lithium to be re-inserted in the subsequent discharge cycle. In this case, the quantity of lithium that is removed cannot be replaced. At the negative electrode, the initial transfer of lithium reduces the potential and induces electrochemical reactions (reduction) with the electrolyte. The reduction 
products are electrochemically inactive species that comprise the solid electrolyte interphase (SEI). This portion of the first charge capacity cannot be accessed in discharge because the reduction products are inactive.

The cell performance forecasts presented in this report assume that the capacity performance for each electrode is the same as in half-cells. The irreversible capacity generated by each electrode must be absorbed by the opposite electrode, as lithium is transferred and current flows during the irreversible process. Allowance for the irreversible capacity forms an important consideration in the capacity balance of the electrodes in a full cell.

\section{Capacity Ratio Considerations}

Practical cell design requires that the capacity of the opposing electrodes be "matched". The electrode pairs have equal active area and must exchange the same capacity in cycling. This is equivalent to completing a material balance for lithium in the electrodes. The positive electrode represents the source of lithium ions for the functioning cell and there must be sufficient negative capacity to absorb the positive capacity (including positive irreversible capacity). The negative electrode is assumed to absorb the positive capacity to form the SEI layer (satisfying the negative irreversible capacity). The balance of remaining positive capacity is assumed to lithiate the negative electrode reversibly.

One of the electrodes may be designed with less capacity than the other, and will limit the capacity of the cell. In this work, calculations assume a positive-to-negative capacity ratio $(\mathrm{P} / \mathrm{N})$ that is slightly less than one, making the cell positivelimited on charge. This choice is intended to provide a slight excess of negative capacity so that lithium can be intercalated into the anode host without forming potentially hazardous (reactive) metallic lithium. In these calculations, the $\mathrm{P} / \mathrm{N}$ ratio is based on the total capacity of the electrodes (irreversible plus reversible capacity).

Sample calculations for a hypothetical, positive-limited, 35 Ah cell are presented below; based on the room temperature electrode performance summarized in Fig. 1.

First, assume a positive reversible capacity of $35 \mathrm{Ah}$. The positive irreversible capacity equals $19.7 \%$ of the reversible capacity $(19.7 \%$ × $35 \mathrm{Ah}=6.90 \mathrm{Ah})$. The total positive capacity is equal to $41.90 \mathrm{Ah}(=35+6.90)$.

This calculation uses excess negative capacity with a $\mathrm{P} / \mathrm{N}$ ratio of 0.909 (equivalent to a $10 \%$ excess negative capacity). Note that this is the ratio of total capacities, including irreversible capacity and reversible capacity. For this cell, the total negative capacity is $46.09 \mathrm{Ah}(=41.90$ / 0.909).

The negative irreversible capacity is equal to $7.7 \%$ of the reversible capacity. Therefore, the negative reversible capacity is $42.79 \mathrm{Ah}(=46.09 \mathrm{Ah} / 1.077)$. The irreversible capacity is $3.30 \mathrm{Ah}(=7.7 \%$ x $42.79 \mathrm{Ah})$. Capacity elements are summarized in Table 2 and compared side-by-side in Fig. 2.

Table 2 Capacity values for baseline 35 Ah cell

\begin{tabular}{rcc}
\hline Capacity in Ah & positive & negative \\
irreversible & 6.90 & 3.30 \\
reversible & 35.00 & 42.79 \\
\cline { 2 - 3 } total & 41.90 & 46.09 \\
irreversible (\% of rev.) & $19.7 \%$ & $7.7 \%$ \\
\hline
\end{tabular}

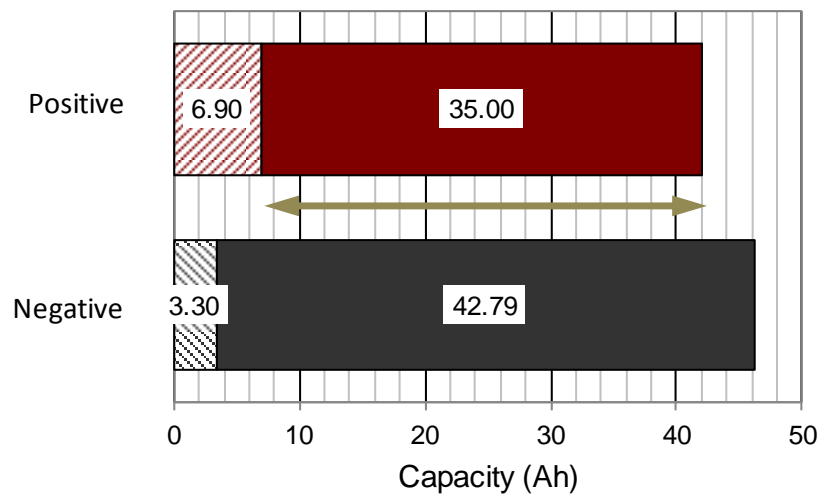

Fig. 2. Capacity Balance in a hypothetical 35 Ah cell.

This hypothetical cell has 35 Ah of reversible capacity in the positive electrode (solid region in Fig. 2). Irreversible capacity is $6.90 \mathrm{Ah}$, illustrated as a hatched region in Fig. 2. This positive electrode will exchange a total capacity of 41.90 $\mathrm{Ah}$ (irreversible + reversible capacity) with the negative electrode in order to become fully charged.

Here, the positive reversible capacity limits the useable window of operation for the negative electrode to the $35 \mathrm{Ah}$ region shown by the arrow in Fig. 2. The limited total positive capacity prevents the negative from becoming fully charged (as expected, since this was the intent of having excess negative capacity). In addition, due to the balance of irreversible capacities, the negative electrode cannot be discharged past the left of the arrow because of the lower limit of positive capacity.

Thus, the maximum state-of-charge for the anode is $90.2 \%$ :

$(6.90+35-3.30) / 42.79=90.2 \%$.

The minimum state-of-charge for the anode is $8.4 \%$ :

$(6.90-3.30) / 42.79=8.4 \%$.

The required weight of electrode active material is computed using the reversible specific capacity for the electrodes (given in Table 1) and the desired cell capacity. 
Results of this calculation appear in Table 3.

Table 3 Electrode active material calculation

\begin{tabular}{rcc} 
& positive & negative \\
\cline { 2 - 3 } reversible capacity $(\mathrm{Ah})$ & 35.00 & 42.79 \\
specific capacity $(\mathrm{mAh} / \mathrm{g})$ & 152.85 & 334.50 \\
active material $(\mathrm{g})$ & 229.0 & 127.9 \\
\hline
\end{tabular}

With the mass of electrode materials established, the voltage of the electrodes (data from Fig. 1) can be determined as a function of the common total capacity as shown in Fig. 3.
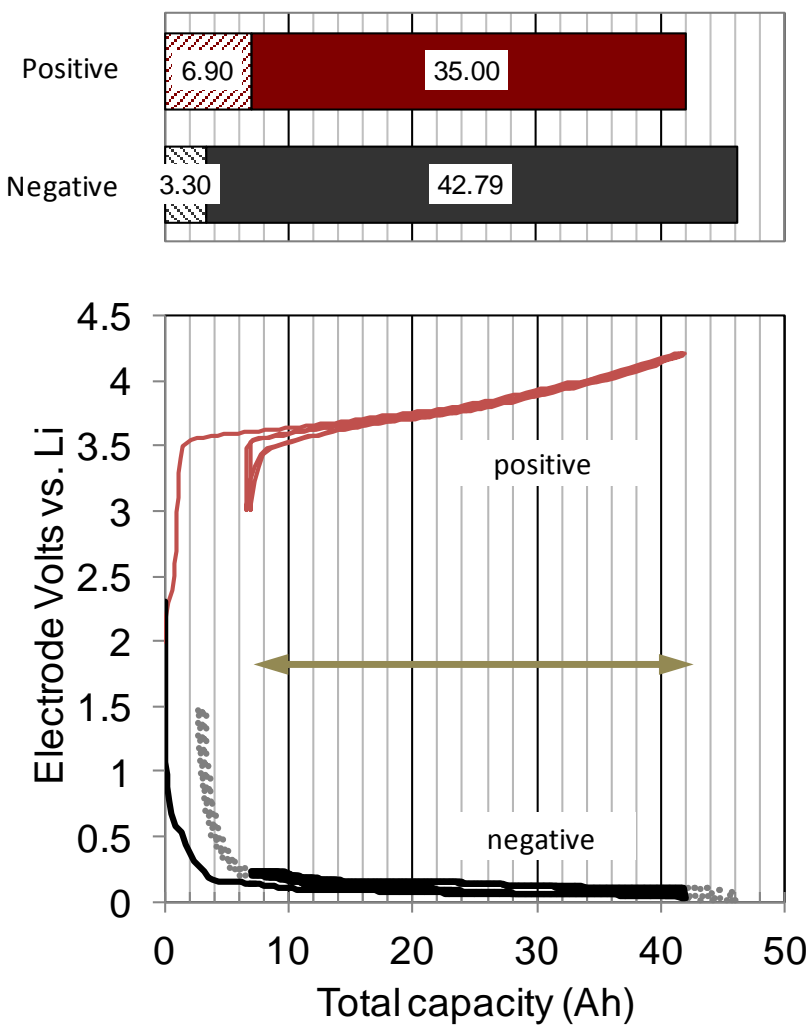

Fig. 3. Electrode voltage profiles in 35 Ah cell.

\section{DISCHARGE PERFORMANCE ESTIMATION}

Here, the process for forecasting cell discharge voltage is described. Laboratory data for single electrodes in half-cells, with lithium metal, is used to predict cell voltage. As seen in Fig. 4, the positive-limited capacity restricts the useful voltage window of the negative electrode. The negative electrode in this example will experience the voltage history illustrated by the solid black line in Fig. 4. The inaccessible negative capacity (illustrated by dotted lines) cannot be utilized. This comparison illustrates how the irreversible capacity and P/N ratio affects the maximum utilization of the individual electrodes. The arrow in Fig. 4 indicates the 35 Ah window for cycling capacity. The limits for the negative electrode state-of-charge in cycling were calculated above to be between
$8.4 \%$ and $90.2 \%$ of the reversible negative capacity.

\section{A. First-charge Capacity}

The first charge capacity accounts for the irreversible capacity of both electrodes. Depending on the P/N ratio, only a portion of the total electrode capacity of the non-limiting electrode can be accessed. The P/N ratio and the balance of the irreversible capacity of the electrodes dictate the useful state-of-charge window of the electrodes. In most cases, this means that the electrodes will not see the full voltage range that was used for half-cell testing. This is illustrated for the negative electrode in Fig. 4. In the initial charge, the total capacity of the positive electrode (41.9 Ah) limits the state-ofcharge of the negative to $90.2 \%$ of its reversible capacity. When the positive electrode is fully discharged, $8.4 \%$ of the total negative discharge capacity remains.

Once the state-of-charge window for both electrodes is determined (by the capacity balance described above) the discharge voltage for the full cell can be estimated.

\section{B. Discharge Capacity}

In this example, the reversible positive electrode capacity is fully utilized on discharge, delivering 35 Ah between electrode potential limits of 4.2 to $3 \mathrm{~V}$ vs. $\mathrm{Li} / \mathrm{Li}+$. Only a portion of the reversible negative capacity is accessed (as discussed above) with state-of-charge ranging from $8.4 \%$ to $90.2 \%$. This $35 \mathrm{Ah}$ portion of negative capacity is illustrated by the solid black line in Fig. 4: now plotted as discharge capacity. The negative electrode has a relatively flat voltage profile in this limited window of operation ( 0.1 to $0.24 \mathrm{~V}$ vs. $\mathrm{Li} / \mathrm{Li}+)$.

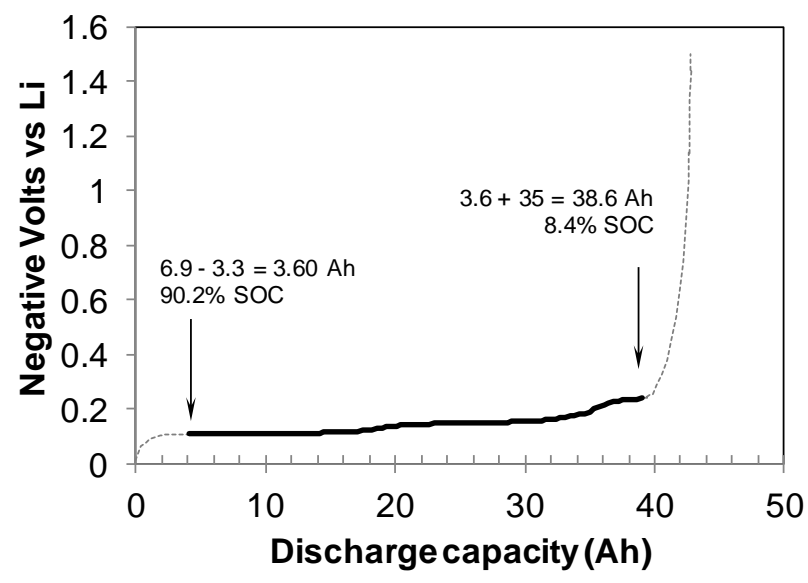

Fig. 4. Discharge capacity window for the anode.

Finally, cell voltage is calculated as the difference between electrode potentials (positive minus negative) over the selected capacity range. Discharge capacity profiles for the electrodes and cell appear in Fig. 5. 


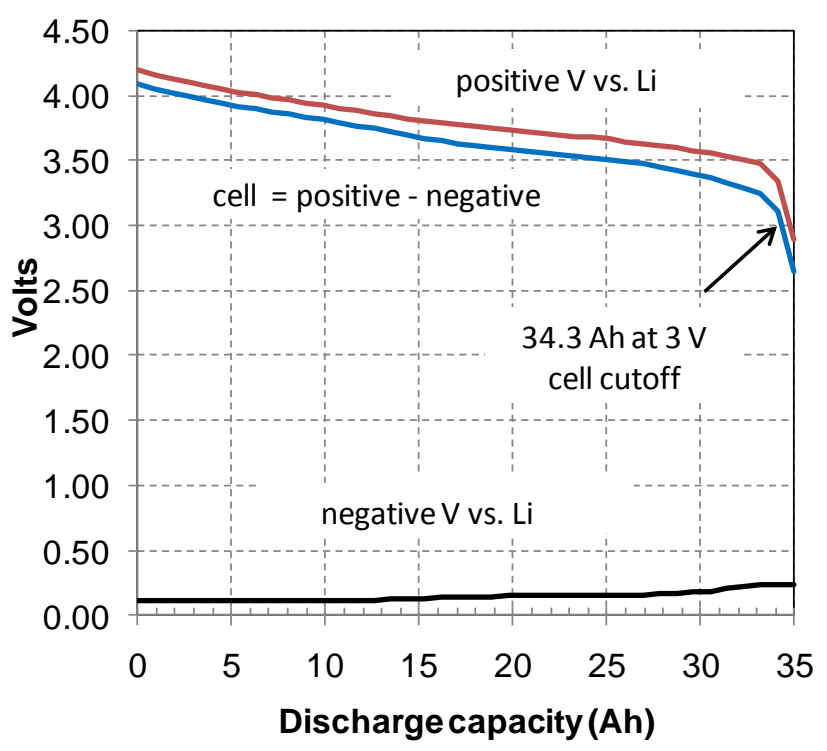

Fig. 5. Electrode and cell potential for the 35 Ah cell.

For this example, the cell was built with a positive electrode capacity selected to deliver $35 \mathrm{Ah}$, based on a positive electrode cutoff voltage of $3 \mathrm{~V}$ vs. $\mathrm{Li} / \mathrm{Li}+$. Factoring in the effect of capacity balance between the positive and negative electrodes, the projected cell voltage reaches a $3 \mathrm{~V}$ cutoff at only 34.3 Ah: $98 \%$ of the 35 Ah target capacity.

For the experimental electrodes presented in this report, positive reversible capacity would need to be increased to $35.75 \mathrm{Ah}$ to achieve $35 \mathrm{Ah}$ at the cell level with a $3 \mathrm{~V}$ cutoff voltage.

\section{CONCLUSION}

The calculation procedures described above provide a means for projecting cell-level discharge voltage performance using laboratory data for individual electrodes. These estimates capture the effect of irreversible capacity, reversible capacity and $\mathrm{P} / \mathrm{N}$ ratio. Results are based on test data for real electrodes in half-cells. The accuracy of such projections depends heavily on the assumptions for electrode makeup. In particular, changes in porosity and electrode loading will have a significant effect on the rate capability of the electrodes. In performing estimates, data at specific loadings and electrode porosity was available.

Results can be integrated to calculate cell energy and, combined with cell mass estimates to predict cell-level specific energy. Estimates for hypothetical prismatic cells are described in [3].

It should be noted that these estimates are based on data collected during the first few cycles of electrode testing. Deterioration of electrode performance over the life of the cell will alter the discharge capacity window. Such effects are not captured by these calculations.

\section{ACKNOWLEDGMENT}

The work presented in this paper was performed in support of NASA's High Efficiency Space Power Systems (HESPS) Project, which is developing advanced lithium-ion cells for future NASA exploration missions under the Enabling Technology Development and Demonstration (ETDD) Program. This work, which began under the Exploration Technology Development Program (ETDP) Energy Storage Project, combines the efforts of industrial partners and contractors to develop aerospace cell designs with enhanced specific energy and safety characteristics [4].

\section{REFERENCES}

[1] Nikkei Electronics Asia -- March 2010, http://techon.nikkeibp.co.jp/article/HONSHI/20100223/180545/

[2] R. Moshtev, B. Johnson, Journal of Power Sources 91 (2000) 86-91.

[3] W. R. Bennett, " Method for Predicting the Energy Characteristics of Li-Ion Cells Designed for High Specific Energy," NASA/TM-2012217407

[4] C. Mercer et al., "Energy Storage Technology Development for Space Exploration", NASA/TM-2011-216964

\section{BIOGRAPHY}

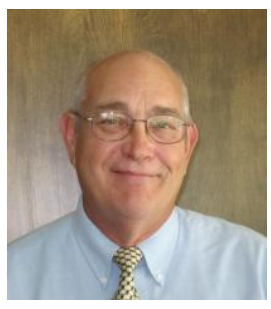

William R. Bennett William Bennett holds a Bachelor's degree in Chemical Engineering. He has worked in the field of electrochemical engineering for over 30 years, building experience in commercial, defense and space applications. Accomplishments have resulted in 7 U. S. Patents and more than 25 technical publications.

Bill has applied the last 11 years of his career supporting a variety of material and battery development projects at the NASA Glenn Research Center (GRC). Bill is currently participating in the development of advanced proton-exchange-membrane (PEM) fuel cell and electrolysis technology for future NASA missions. Current engineering interests include system analysis and water management strategies for PEM fuel cells. 